\title{
Silicon self-assembled growth of quantum dots grown on corning glass (7059) substrate
}

\author{
Samsudi Sakrani*, Lim Qiao Jie and Imam Sumpono \\ Ibnu Sina Institute for Fundamental Science Studies, Universiti Teknologi Malaysia, 81310 UTM Skudai Johor, Malaysia. \\ ${ }^{\star}$ To whom correspondence should be addressed. E-mail: samsudi@dfiz2.fs.utm.my
}

Received 15 March 2007

http://dx.doi.org/10.11113/mjfas.v3n1.24

\section{ABSTRACT}

Silicon quantum dots have been grown on corning glass (7059) substrate using a self-assembly method of physical vapour deposition. The samples were fabricated at sufficiently low sputtering rate and varying experimental conditions. Apparently, the onset of nucleation took place during the first $\mathbf{5}$ minutes of deposition, followed by a further growth of stable islands known as quantum dots, with the measured radii comparable to the predicted values. Other measurement results confirmed the existence of these dots, including the bandgap energy $\sim 1.80 \mathrm{eV}$ from $\mathrm{PL}$ and a $2 \%$ at. silicon from EDX and possible amorphous from XRD. The nucleation parameters were predicted as follows: Free energy change per unit volume $\Delta G_{v}=2.64 \times 10^{4} \mathrm{Jmol}^{-1}$; Surface energies per unit area, $\gamma_{L N}=1.48 \mathrm{Jm}^{-2}, \gamma_{N S}=20.0-90.0$ $\mathrm{Jm}^{-2}$ and $\gamma_{L S}=0.42 \times 10^{-2} \mathrm{Jm}^{-2}$; Critical energies $\Delta G^{*}=7.53 \times 10^{-17}-8.31 \times 10^{-14} \mathrm{~J}$; Critical radii $r^{*}=9.0-97.0 \mathrm{~nm}$. This experimental evidence strongly support the early stage growth model of silicon quantum dot deposited on corning glass substrate.

| Silicon | Self-assembled Growth | Quantum Dot | Corning (7059) Glass |

\section{Introduction}

A great interest in the application quantum dots is currently found in the fields of science and engineering, in which nanofilms become the ground of their elementary base. The general description of nanofilm formation has been overwhelmingly emphasized by many nanotechnologists during the recent years [1], but an in depth understanding of the phenomena is still in slow progress, mainly due to lack of information on the very early stage of nanofilm, called nucleation. In general, nanofilm nucleation is defined as the creation of small clusters or nuclei that responsible for film growth on selected substrate, a phenomena known as heterogeneous nucleation. So far, no rigorous theory of heterogeneous new phase nucleation on a surface has yet been developed, except those on classical or capillary nucleation theory $[2,3]$. It is the purpose of this paper to investigate suitable approaches on the issue, with highlight on silicon quantum dots.

Rapid advances in silicon circuit fabrication and design are paving the way for creation of disruptive technologies that can significantly improve the performance of everyday appliances such as computers, cell phones and digital cameras. Miniature silicon device that drive many such appliances are expected to become smaller and faster. 
Features that are currently $50 \mathrm{~nm}$ large will become as small as $10 \mathrm{~nm}$ by a decade and the speed will increase more than four times. Silicon quantum dot is the possible candidate for tiny devices, such as single-electron transistors [4] and biological sensors [5].

\subsection{Nucleation and Volmer-Weber Growth}

In order to understand the growth mechanism, an equilibrium study of dislocation-free island formation has been carried out by Daruka and Barabási [6]. The results are summarized in the phase diagram shown in Fig. 1, which presents the possible growth modes in functional relationship to the number of monolayers deposited $(\vartheta)$ and the lattice mismatch $(\varepsilon)$. The phase diagram distinguishes a number of different growth mechanisms, including twodimensional layer-by-layer growth or Frank-van der Merwe (F-M), Stranski-Krastanov (S-K), and VolmerWeber $(\mathrm{V}-\mathrm{W})$ growth. These growth modes generate stable islands, whereas three different kinds of ripening which may or may not include a two-dimensional layer (frequently called the wetting layer) are possible. The phase diagram demonstrates that in order to achieve stable and controllable S-K or V-W growth, for which uniform small islands may result, it is necessary to employ semiconducting crystal systems that include a large amount of strain (i.e., work far out along the horizontal axis in this diagram), and the deposited material must not exceed a certain critical coverage.

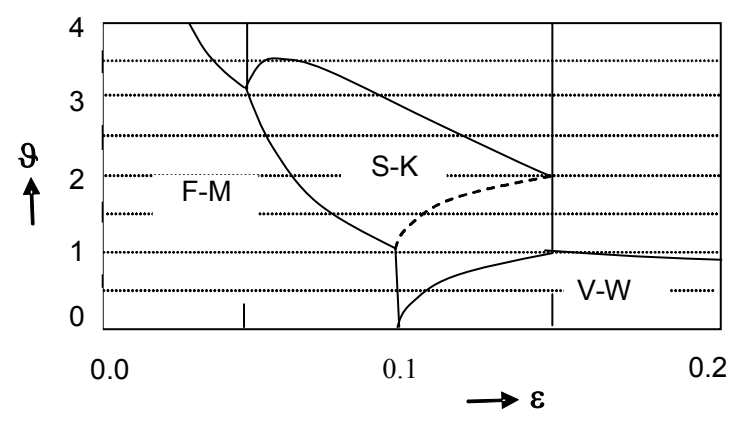

Figure 1. Equilibrium phase diagram for self-assembled quantum dot formation [6].

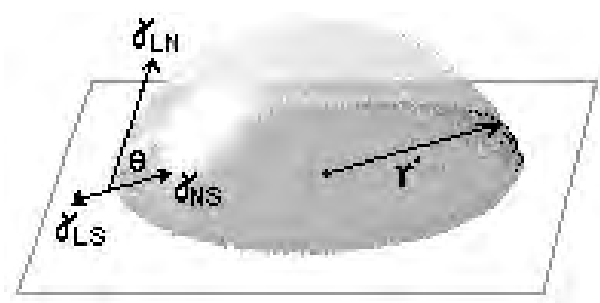

Figure 2. Dome-like shape of a nucleus, showing the related parameters

For the case of $\mathrm{V}-\mathrm{W}$, it appears that growth takes place in the region $0.1<\varepsilon<0.2$ over the monolayer $\vartheta=0.0-$ 1.0. However, some selected non-crystalline and amorphous materials including semiconductors tend to fulfill the requirement of $\mathrm{V}-\mathrm{W}$ growth if the surface parameters are taken into consideration. A liquid to solid phase transition is often initiated by a nucleation process, manifested by the appearance of nuclei of the new phase. The process is made possible by diffusion of atoms or molecules that aggregate to form the nuclei of liquid, with the size of these nuclei increases with time and at the end of the process a new homogenous solid is formed, i.e. 3dimensional islands with increased volume. A stable nucleus shown in Fig. 2 is governed by three surface energies per unit area, $\gamma$ and contact angle, $\theta$ according to the relationship:

$$
\gamma_{L S}=\gamma_{L N} \cos \theta+\gamma_{N S}
$$

Two conditions of film growth are possible:Firstly, if $0<\cos \theta<1$ (for $\theta<90^{\circ}$ ); then $\gamma_{L S}<\gamma_{L N} \cos \theta+\gamma_{N S}$. This solution gives the wetting condition between the nucleus and substrate, thus forming a dome-like shape of nanofilm due to F-M growth (Fig. 2). The film atoms are more strongly bound to each other than to substrate. 
Secondly, if $-1<\cos \theta<0$ (for $\theta>90^{\circ}$ ), then equation (1) is fulfilled. In such a case the nucleus is being pulled inside to be in a non-wetting oval shape following V-M growth mode. This is normally occurs in the deposition of metals on dielectrics. Similar phenomena could be adapted for silicon nanofilm if a suitable substrate is chosen.

\subsection{Theoretical Consideration}

It is assumed that the atoms/vapour are initially coalesced to each other followed by agglomeration, forming small clusters similar to nuclei of specific sizes, and the process is proceeded with further increase of sizes up to their critical conditions. These unstressed nuclei are in spherical shape, each of which comprising of several thousand of atoms depending on the nature of bonding. Essentially Si crystallites with zincblend structure shall accommodate less number of atoms if compared to amorphous Si within the same volume of spherical nuclei. In a situation where nucleation is heterogeneous, the intervention of new interfaces must be taken into consideration: those between the solid nucleus-substrate surface, liquid-solid nucleus and liquid-substrate surface, each of which is shown by the respective surface energies per unit area, $\gamma_{N S}, \gamma_{L S}$, and $\gamma_{L N}$ (Fig. 2).

From the thermodynamic point of view, a change in the free enthalphy, $\Delta G$ is associated with nucleation and with these four process: Firstly, creation of new volume of the nucleus (negative); secondly existence of surface area of liquid-nucleus (positive), thirdly existence of surface area of liquid-nucleus (positive) and lastly the energy lost at liquid-substrate interface (positive. Thus,

$$
\Delta G=\alpha^{3} r^{3}\left(-L_{f} \frac{\Delta T}{T_{m}}\right)+\alpha_{1} r^{2} \gamma_{L S}+\alpha^{2}\left(r^{2} \gamma_{N S}-r^{2} \gamma_{L N}\right)
$$

where, $\alpha_{1}=2 \pi(1-\cos \theta), \alpha_{2}=\pi \sin ^{2} \theta, \alpha_{3}=\pi\left(2-3 \cos \theta+\cos ^{3} \theta\right) / 3, \Delta G_{v}=L_{f}\left(\Delta T / T_{f}\right)$ is the energy change per unit area of the nucleus and has positive values if $T>T_{m}$, with $T_{m}$ being the temperature of the transition (solidification temperature). Under these conditions, applying the Gibbs-Duhem principle, the new phase is not stable. Since $\gamma$ is a positive quantity, $\Delta G_{v}$ is then positive: the number of nuclei of the new phase are not stable and do not tend to grow. On the contrary, if $T<T_{m}$, is negative, the new phase (solid) is stable. There are values of $r$ for which $\Delta G_{v}<0$, and the corresponding nuclei will tend to stabilize the new phase since their formation reduces the free enthalpy of material. When $T<T_{m}(\Delta T>0), \Delta G_{v}$ has a maximum for $r=r^{*}$. For $r>r^{*}$, the formation of nuclei of increasing size can results in stabilization of the solid phase since $\Delta G_{v}$ decreases and even becomes negative. Both the critical energy, $\Delta G^{*}$ and critical radius, $r^{*}$ are obtained as,

$$
\begin{gathered}
\Delta G^{*}=\frac{4\left(\alpha_{1} \gamma_{L N}+\alpha_{2} \gamma_{N S}-\alpha_{2} \gamma_{L S}\right){ }^{\beta} T_{m}^{2}}{27 \alpha_{3}^{2} \Delta H_{f}^{2} \Delta T^{2}} \\
r^{*}=\frac{-2\left(\alpha_{1} \gamma_{L N}+\alpha_{2} \gamma_{N S}-\alpha_{2} \gamma_{L S}\right) T_{m}}{3 \alpha_{3} \Delta H_{f} \Delta T}
\end{gathered}
$$

$\Delta G^{*}$ is equivalent to the potential barrier that must be lowered for nuclei of critical size $r^{*}$ to be formed more easily $[1,2]$. It seems that from equation (2) the more important supercooling is, the more this barrier is lowered. The Volmer thermodynamic model shows that a complete phase transition by nucleation is only possible if the liquid is supercooled. In fact, nucleation is initiated with lower supercooling because the impurities present in the liquid phase, even in low concentration, induce nucleation, normally referred to heterogeneous nucleation. 


\section{Experimental}

The samples were fabricated using a magnetron sputtering system at sufficiently low deposition rate of $2.7 \times 10^{-2}$ $\mathrm{nms}^{-1}$ and varying experimental conditions: Chamber pressure $10^{-2} \mathrm{Torr}$, Heater temperature $100-400^{\circ} \mathrm{C}$, fixed argon flow rate $5 \mathrm{sccm}$, and deposition times varied between 5-20 minutes. The principal process was based on high energetic burial of target materials previously removed by positive ion bombardment within the plasma region. This high-speed particle transportation may contributes to the initial growth of quantum dot on selected substrate surface. The samples were analysed using photoluminescence, EDX and XRD. A theoretical investigation was done using the classical approach of heterogeneous nucleation in which the net energy change required to form a spherical nucleus is equivalent to the summation of energy terms associated with four different interfacial interactions.

\section{Result and Discussion}

The analysis obtained from AFM revealed the early formation of nuclei resembling quantum dots of varying sizes and shapes (Fig. 3) formed at different deposition times. The dots in Fig. 3a) appears to be in dome shapes of sizes between $60-90 \mathrm{~nm}$, which are slightly different from that of Fig. 3b) in terms of their pyramidal shapes and larger sizes. Photoluminescence curves in Fig. 4 show the peaks centered at $\lambda=692 \mathrm{~nm}$ and the associated band gap energy of the dots, $E_{g} \sim 1.80 \mathrm{eV}$ ( $>1.1 \mathrm{eV}$ of the bulk silicon), the result which gives an indicative of size reduction in silicon quantum dots. Analysis from EDX (Fig. 4) and Grazing incident X-ray scattering or GIXRS (Fig. 5) reveal a $\%$ increase of Si contents and non-crystalline nature of the samples, respectively. It is clear that, the later result is dominated by the noises and may suggest the existence of amorphous quantum dot. All these experimental evidence strongly support the formation of silicon quantum dot deposited on corning glass substrate.

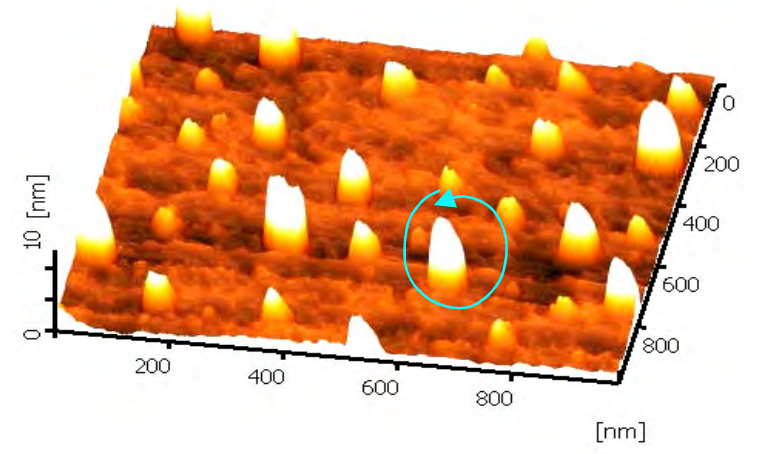

a)

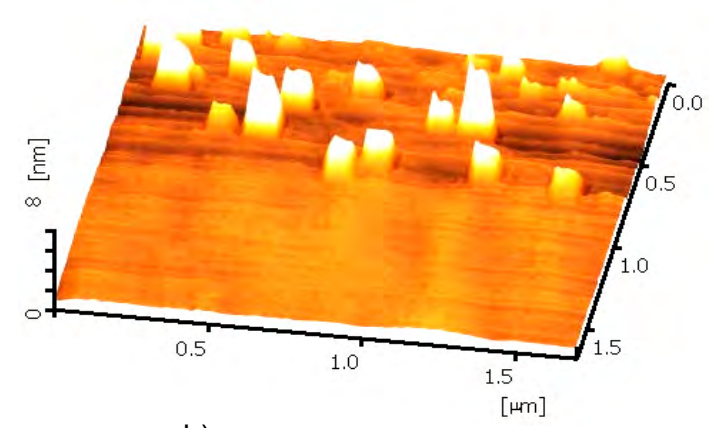

b)

Figure 3. Silicon quantum dots formed after a) 5 mins. and b) 15 mins. of depsosition 
Simulation works have been performed using equations (2) to (4) by substituting appropriate parameters of enthalpy of fusion, $H=3.96 \times 10^{4} / \mathrm{mol}$; melting temperature, $T_{m}=1683 \mathrm{~K}$; substrate temperature, $T=563 \mathrm{~K}$; undercooling temperature, $T_{m}-T=1120 \mathrm{~K}$, atomic volume, $v_{o}=12.1 \mathrm{~cm}^{3} / \mathrm{mol}$ and contact angle, $\theta=70^{\circ}$. The growth parameters were computed over the specific ranges, namely $\gamma_{N S}=20-90 \mathrm{Jm}^{-2}, \gamma_{L S}=0.42 \times 10^{-2} \mathrm{Jm}^{-2} ; \Delta G^{*}=$ $7.53 \times 10^{-17}-8.31 \times 10^{-14} \mathrm{~J} ; r^{*}=9.4-97.4 \mathrm{~nm}$, with the best fit values of $\Delta G_{v}=2.64 \times 10^{4} \mathrm{Jmol}^{-1}$ and $\gamma_{L N}=1.48 \mathrm{Jm}^{-2}$. All these results are tabulated in Table 1.

Figures 6 and 7 show the variations of both $r^{*}$ and $\Delta G^{*}$ with $\gamma_{N S}$ and $\theta$. The graph in Fig 6 clearly indicate the increasing modes of $r^{*}$ with $\gamma_{N S}$ in (straight line 1) and $\Delta G^{*}$ with $\gamma_{N S}$ (curve 2). In contrast, the decreasing modes of $\Delta G^{*}$ and $r^{*}$ with $\theta$ (Fig. 7) are indicated by the curves 3 and 4 respectively, as described earlier [7]. These results strongly suggest that both critical parameters are the functions of surface energy at the nucleus-surface interface and contact angle between the nucleus and substrate. Figure 3 show silicon quantum dots observed using AFM formed after i) 5 and ii) 15 minutes of deposition. A further investigation of the dot's cross section in Fig. 8 (circled in blue of Fig. 3) confirms the formation of a dome shape, with $r^{*}=37.4 \mathrm{~nm}$. By substituting $r^{*}$ into equation (3) the following computed parameters were obtained: $\gamma_{N S}=48 \mathrm{~J} / \mathrm{m}^{2}, \gamma_{L S}=0.42 \mathrm{~J} / \mathrm{m}^{2}$ and $\Delta G^{*}=$ $5.67 \times 10^{-15}$ which were comparable to those measured elsewhere [8,9]. The figures in bracket of Table 1 show these values.

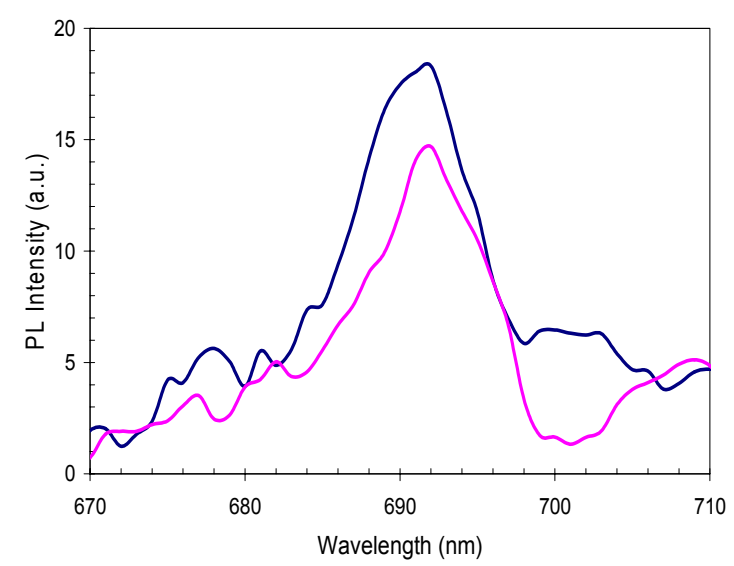

Figure 4. PL intensities for selected samples

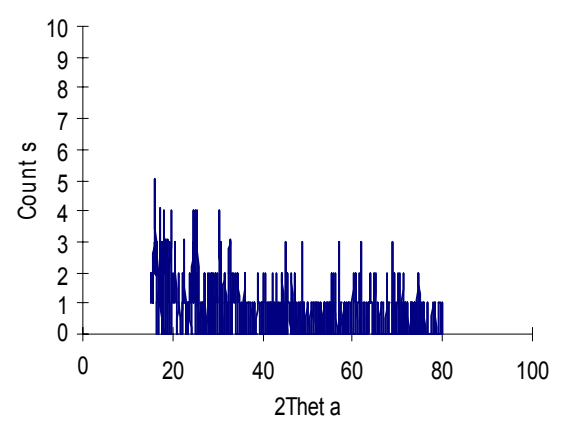

Figure 5. GIXRS patterns 
Table 1. Computed growth parameters

\begin{tabular}{|l|c|c|}
\hline \multicolumn{1}{|c|}{ PARAMETER } & COMPUTED & REFERENCE \\
\hline $\begin{array}{l}\text { Surface energy/unit area at liquid- } \\
\text { nucleus interface, } \gamma_{L N}\left(\mathrm{~J} / \mathrm{m}^{2}\right)\end{array}$ & 1.48 & $1.44-1.75[8]$ \\
\hline $\begin{array}{l}\text { Surface energy/unit area at nucleus- } \\
\text { substrate interface, } \gamma_{N S}\left(\mathrm{~J} / \mathrm{m}^{2}\right)\end{array}$ & $\begin{array}{c}10.0-100.0 \\
(48.0)\end{array}$ & $0.30-0.75[9]$ \\
\hline $\begin{array}{l}\text { Surface energy/unit area at liquid- } \\
\text { surface interface, } \gamma_{L S}\left(\mathrm{~J} / \mathrm{m}^{2}\right)\end{array}$ & $(0.42)$ & - \\
\hline $\begin{array}{l}\text { Free energy change } / \mathrm{unit} \text { volume, } \\
\Delta G_{v}\left(\mathrm{~J} / \mathrm{mol}^{2}\right)\end{array}$ & -26352.9 & - \\
\hline $\begin{array}{l}\text { Critical radius, } r^{*}(\mathrm{~nm}) \\
\begin{array}{l}9.4-97.4 \\
(37.4)\end{array}\end{array}$ & $\begin{array}{c}7.53 \times 10^{-17}-4.85 \times 10^{-14} \\
\left(5.67 \times 10^{-15}\right)\end{array}$ & - \\
\hline $\begin{array}{l}\text { Critical energy, } \Delta G^{*} \\
(\mathrm{~J})\end{array}$ & & \\
\hline
\end{tabular}

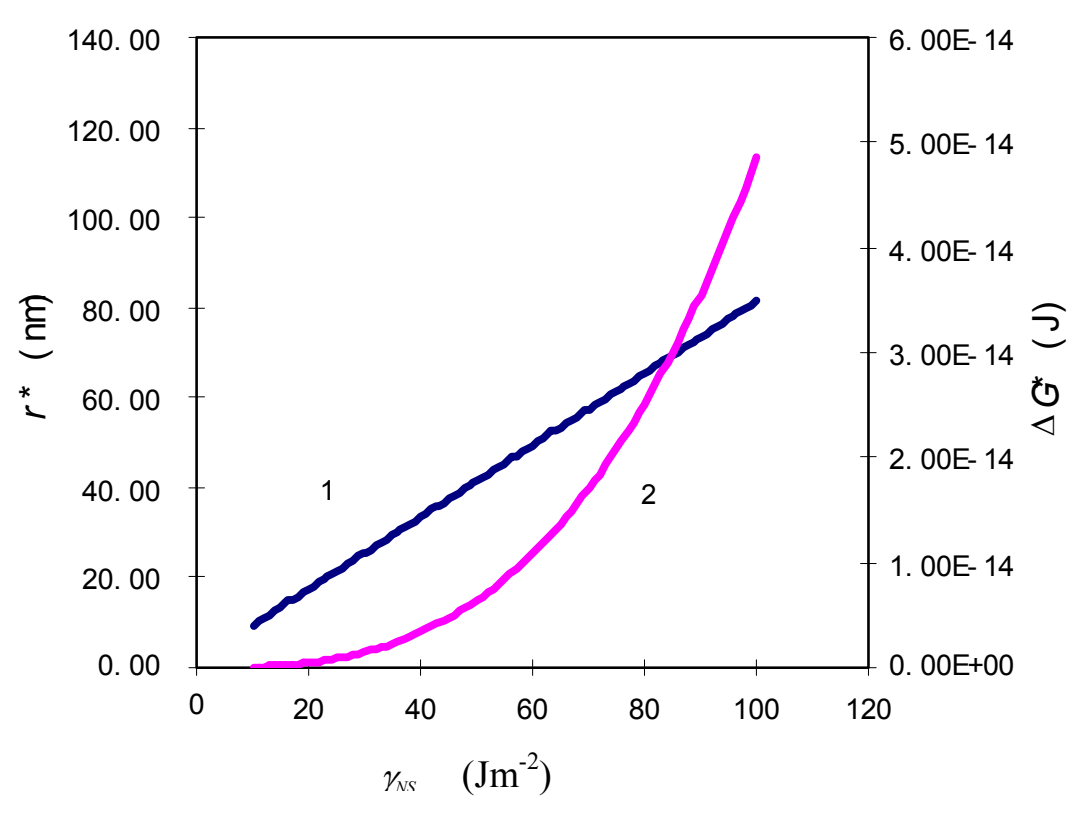

Figure 6. Variations of $r^{*}$ and $\Delta G^{*}$ as a function of energy per unit area, $\gamma_{N S}$ 


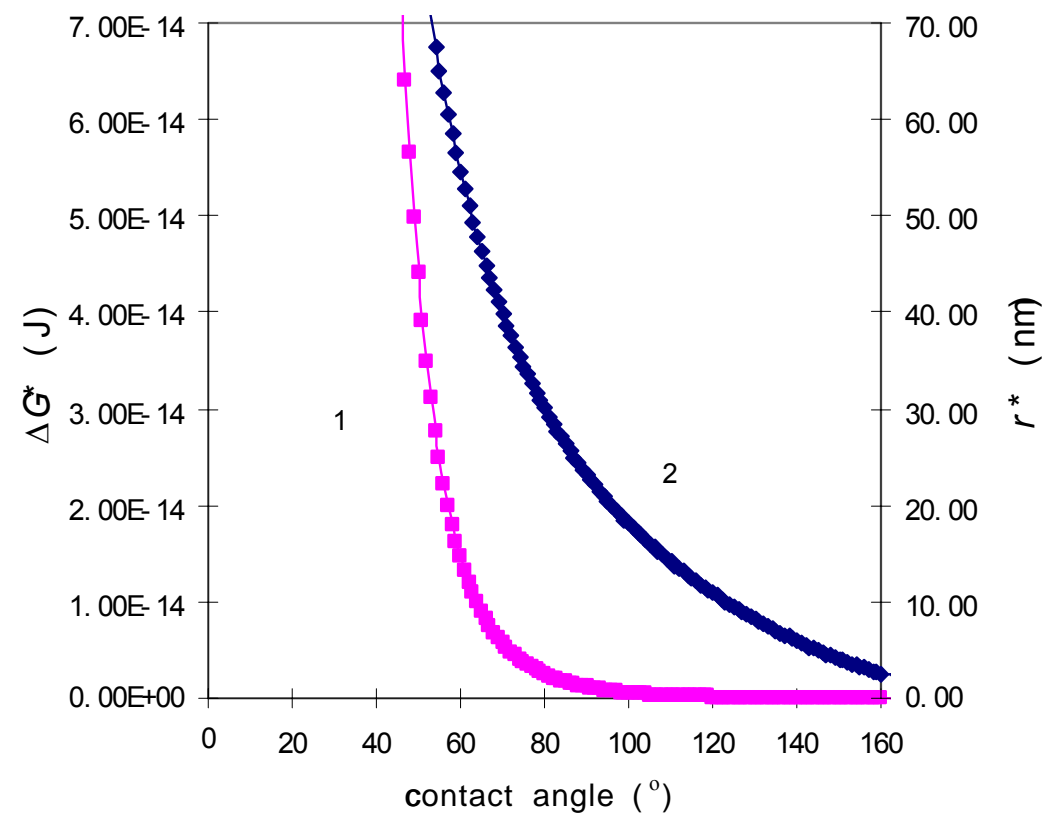

Figure 7. Variations of $r^{*}$ and $\Delta G^{*}$ as a function of contact angle, $\theta$

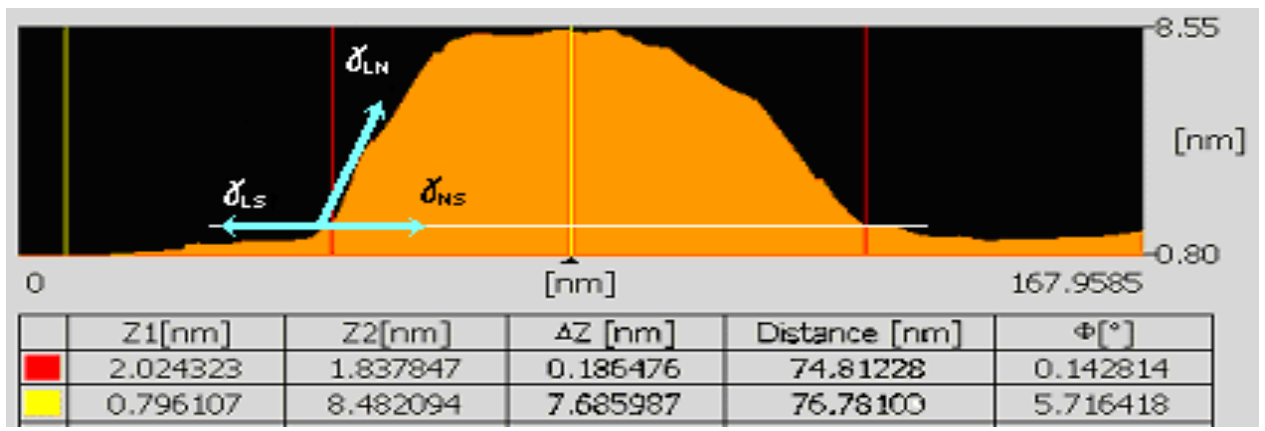

Figure 8. Cross section of a dome shape quantum dot

A description of the formation of Si quantum dots was then established based on the modification of VolmerWeber growth model. Accordingly, the growth satisfies the surface energy equation (Young equation), $\gamma_{L S}<\gamma_{L N}$ $\cos \theta+\gamma_{N S}$ and expecting the formation of stable nuclei, preferentially in oval shapes at the end of critical conditions. However, a dome shape dot was observed (Fig. 8) just like the F-M mode growth. It is believed that, from the computed surface energies at the nucleus-surface interface, a range of $\gamma_{N S}=20-90 \mathrm{Jm}^{-2}$ were developed during the growth, thus establishing stronger binding between the silicon atoms and substrate surface if compared to their neighboring inter atomic interactions. This provides the required interface wetting under the condition of $\vartheta<1$ (Fig. 1). It is therefore recommended that, the original growth following Volmer-Weber mode shall be 
modified for the case of amorphous material deposited onto amorphous substrate, in particular the fabrication of silicon quantum dot on the surface of corning (7059) glass.

\section{Conclusions}

The early stage of silicon quantum dot formation can be well described using a modification of the growth mode, with parameters predicted from the theory. Under condition that Young equation is fulfilled this growth occurred during the early stage of deposition and did not show the predicted non-wetting oval nucleus. Instead, a wetting dome-like shape was formed and establishes the basic quantum dot structures. This self-assembly method can be applied for the fabrication of quantum devices, such as single-electron transistor and nanobiosensor.

\section{Acknowledgement}

The authors would like to thank the Government of Malaysia for providing financial support under the IRPA grant No. 09-02-06-0157-SR0013/06-02 (vot 74542).

\section{References}

[1] Kukushkin, S. A. and Osipov, A. V., Nucleation Kinetics of Nanofilms, H. S. Nalwa, Eds., Encyclopedia of Nanoscience and Nanotechnology, Am. Sci. Pub., 8 (2004) 113-136.

[2] Wagendristel, A. and Wang, Y., An Introduction to Physics and Technology of Thin Films, World Scientific Publ., Singapore, (1994) 30-39.

[3] Schmezer, J., Ropke, G. and Priezzhev, V. B. Eds., Nucleation Theory and Applications, JINR, Dubna, 2002.

[4] Hashim, U., Rasmi, A. and Sakrani, S., SOI - Based nanowire single-electron transistors: design, simulation and process development, Int. J. Mat. Sci \& Simul (IJMSS), 1. Iss. 1 (2007) 18-32.

[5] Vo-Dinh, T., Nanobiosensors: probing the sanctuary of individual living cells, J. Cell Biochem., 39 (suppl.), (2002) 154-161.

[6] Daruka, I. and Barabasi, A-L., Dislocation-Free Island Formation in Heteroepitaxial Growth: A Study at Equilibrium, Physical Review Letters, 79, No.19, (1997) 3708-3711.

[7] Sakrani, S., Lim, Q. J. and Wahab, Y., The Formation of Ultra Small Clusters Predicted Using a Capillary Model of Nucleation, J. Fundamental Sciences, 1, No.1, (2005) 21-31.

[8] Eaglesham, D.J., White, A.E., Feldman, L.C., Moriya, N. and Jacobson, D.C., Equilibrium Shape of Silicon, Physical Review Letters, 70(11) (1993) 1643-1646.

[9] Shand, E. B., Note on Surface Energy of Glass, J. Am. Ceramic Soc., 47, Iss. 2, (1964) 102-119. 